\title{
Tecnologías Digitales en el Aprendizaje- Servicio para la Formación Ciudadana del Nuevo Milenio
}

\section{(Digital Technologies in Service Learning for the Formal Education of the New Millennium Citizenship)}

\author{
Beatriz Sandia Saldivia \\ Jonás Montilva Calderón \\ Universidad de Los Andes (Venezuela)
}

DOI: http://dx.doi.org/10.5944/ried.23.1.24138

\section{Cómo referenciar este artículo:}

Sandia Saldivia, B., y Montilva Calderón, J. (2020). Tecnologías Digitales en el Aprendizaje-Servicio para la Formación Ciudadana del Nuevo Milenio. RIED. Revista Iberoamericana de Educación a Distancia, 23(1), pp. 129-148. doi: http://dx.doi.org/10.5944/ried.23.1.24138

\section{Resumen}

La Sociedad Digital, caracterizada por la aceleración tecnológica, la producción de nuevos conocimientos, la disponibilidad y acceso a la información y la comunicación, interacción y colaboración a través de Internet, obliga a replantear la formación universitaria orientándola hacia el desarrollo de actitudes y competencias necesarias para una formación ciudadana adaptada a las necesidades de esta nueva sociedad. Este trabajo presenta los resultados de un análisis documental de las competencias, habilidades, valores y actitudes que caracterizan la ciudadanía del nuevo milenio; además de cómo el enfoque educativo de aprendizaje-servicio juega un papel preponderante en la formación ciudadana promoviendo aquellas competencias que son pertinentes y necesarias al proporcionar oportunidades para conectar el currículo con la comunidad y desarrollar habilidades y actitudes de participación cívica. Así mismo, se presenta un modelo de referencia para la comprensión de los espacios virtuales para el aprendizaje-servicio (EVApS). Este modelo fue elaborado usando un enfoque sistémico que concibe a este tipo de tecnologías como sistemas abiertos, intencionales, formados por conjuntos de componentes heterogéneos, los cuales se interrelacionan para proporcionar a sus usuarios una funcionalidad que apoya los procesos de enseñanza y aprendizaje orientados al aprendizaje-servicio. Este modelo ayuda a comprender y diseñar EVApS de manera integral y sistemática; así como a identificar el conjunto de tecnologías digitales que pueden ser utilizadas para apoyar el desarrollo de competencias propias del aprendizaje-servicio durante la formación de la ciudadanía del nuevo milenio. 
Palabras clave: aprendizaje-servicio; espacios virtuales de aprendizaje; tecnologías de la información y de la comunicación; educación ciudadana; proceso de aprendizaje.

\begin{abstract}
The Digital Society, characterized by a fast-changing technological development, the production of new knowledge, the availability and access to information, and the communication, interaction and collaboration through the Internet, requires rethinking the university education of young people, orienting it towards the development of attitudes and competencies which are necessary for citizen education adapted to the needs of this new society. This paper presents the results of a documental analysis of the competencies, skills, values and attitudes that characterize citizens of the new millennium. It also describes how the educational approach of service-learning plays a preponderant role in the training of this kind of citizen, promoting those competencies that are relevant and necessary to provide opportunities to connect the curriculum with the community and develop civic participation skills and attitudes. A reference model for the understanding of virtual spaces for servicelearning (EVApS) is also presented in this paper. It was developed using a system approach that conceives this type of technology as open and intentional systems, which are composed of a set of heterogeneous components. These components are interrelated in order to provide its users with a required functionality and support to the teaching and learning processes oriented to service learning. This model helps to understand and design EVApS in an integral and systematic way. It also identifies a set of digital technologies that can be used to support the development of competencies specific to service learning with a view to training the citizens of the new millennium.
\end{abstract}

Keywords: service learning; virtual learning spaces; information and communication technologies; citizenship education; learning process.

La Sociedad Digital o Sociedad 3.0, donde las innovaciones tecnológicas han facilitado el desarrollo de escenarios digitales para la construcción del conocimiento, la disponibilidad y acceso a la información, la comunicación, interacción y colaboración en red, así como la posibilidad de entender y enfrentar la complejidad, es una sociedad que debido al aceleramiento de los procesos ha generado nuevos modelos productivos y sociales. Es una sociedad donde existe una alta conectividad entre personas, información y conocimiento; donde se promueve la sostenibilidad y se fortalece la reciprocidad a través de una economía interdependiente (Van den Hoff, 2013). Es una sociedad en la que la vida de la ciudadanía digital se desarrolla en los espacios y tiempos construidos en la Web 3.o (Sandia, Arnal, Moreno, Mujica, Hernández y Páez, 2018). Los espacios virtuales desempeñan en esta sociedad un papel fundamental al permitir que la ciudadanía se comunique, interactúe, coopere y colabore remotamente a través de la WWW.

En el sector educativo universitario, los espacios virtuales de aprendizaje son ampliamente utilizados en diferentes modalidades de aprendizaje, incluyendo la 
modalidad presencial, la virtual y la combinada. Sus usos, ventajas, desventajas e impacto en los procesos de educación universitaria han sido ampliamente estudiados por varios investigadores: (Alves, Miranda y Morais, 2016, 2017; Herrera Mosquera, 2017; Kurt y Tingoy, 2017).

En este artículo se analizan, en primer lugar, las competencias, habilidades, valores y actitudes que caracterizan, según diferentes autores, la ciudadanía del nuevo milenio, ese individuo que colectivamente constituye una parte fundamental de la Sociedad Digital. Luego se discute el papel que juega el aprendizaje-servicio en la formación de este ciudadano y se identifican aquellas competencias que son pertinentes y necesarias en la formación ciudadana. Finalmente, se presenta un modelo de espacios virtuales para el aprendizaje-servicio que concibe a este tipo de tecnologías como sistemas abiertos, intencionales, formados por un conjunto de componentes heterogéneos, que se interrelacionan para proporcionar a sus usuarios una funcionalidad que apoya los procesos de enseñanza y aprendizaje orientados a la prestación de servicios comunitarios.

Los resultados relacionados con las competencias que los ciudadanos y ciudadanas del nuevo milenio deben desarrollar y, en particular, aquellas asociadas al aprendizaje-servicio, son producto de una investigación documental de tipo expositiva, en la cual se analizó un conjunto de referencias relevantes al aprendizajeservicio y a la formación ciudadana en la Sociedad 3.o. A partir de este análisis se estableció un conjunto de competencias que caracterizan al ciudadano del nuevo milenio y que pueden ser usadas en la definición de objetivos de aprendizaje en cualquier proyecto de aprendizaje-servicio. El segundo resultado es un modelo de referencia que describe, desde una perspectiva sistémica, diferentes aspectos de los espacios virtuales de aprendizaje y, en particular, de aquellos orientados al aprendizaje-servicio. Este modelo identifica, además, un conjunto de tecnologías digitales que pueden ser utilizadas para apoyar el desarrollo de competencias propias del aprendizaje-servicio. Para la construcción de este modelo se emplearon métodos y técnicas provenientes de la Ingeniería del Software y los Sistemas Complejos (Serrano Zaneti, 2014). El método ontológico-sistémico propuesto por Montilva y Montilva (2018) fue utilizado para especificar los diferentes aspectos de este tipo de espacio virtual a través del uso de técnicas de modelado de arquitecturas de software y modelado sistémico.

\section{FORMACIÓN PARA LA CIUDADANÍA DEL NUEVO MILENIO}

Nuestra realidad social actual nos obliga a pensar en la necesidad de formar ciudadanos y ciudadanas del mundo para un nuevo milenio, con actitudes, habilidades y destrezas orientadas, principalmente, a reconocerse como seres humanos universales, cuyo accionar se fundamenta en la libertad, la autonomía y la responsabilidad; seres capaces de aprender a enfrentar la incertidumbre y desarrollar un pensamiento crítico, dispuestos a innovar y generar nuevo conocimiento, tal y 
como lo señala Morin (2002), en su libro "Siete Saberes Necesarios para la Educación del Futuro".

En este orden de ideas, formar para la ciudadanía global implica que los individuos comprendan la posibilidad de una "identidad colectiva" con un conocimiento profundo de los problemas mundiales y de los valores universales, que posean competencias comunicacionales y habilidades para el trabajo colaborativo, capaces de enfrentar y dar soluciones a esos problemas, con un pensamiento crítico, creativo y sistémico, y luchadores por el bien colectivo (UNESCO, 2016).

De igual manera, la UNESCO (2017) en la "Agenda Global de Educación 2030", plantea que, para un desarrollo sostenible global, la educación debe enfocarse en formar individuos capaces de reflexionar sobre sus propias acciones y su repercusión en todos los ámbitos. Se requiere de individuos generadores del cambio con conocimiento, habilidades, valores y actitudes para promover una vida sostenible, pacífica, próspera y equitativa para todos.

En una investigación desarrollada por Pearson, Nesta y la Universidad de Oxford en el 2017, denominada: "El futuro de las habilidades: el empleo en 2030", en la que se analizan el cambio tecnológico, la globalización, el crecimiento demográfico, la sostenibilidad del medio ambiente y el crecimiento de la desigualdad, entre otras tendencias que influyen en las habilidades requeridas para el trabajo en el futuro cercano, señala que existe un énfasis importante en las habilidades interpersonales, así como en las habilidades de alto nivel cognitivo tales como la originalidad, la fluidez de ideas, el aprendizaje activo y las estrategias de aprendizaje (Bakhshi, Downing, Osborne y Schneider, 2017).

Por otro lado, el Banco Mundial, en el reporte World Development Report 2019: The Changing Nature of Work (World Bank, 2019), presenta un análisis de cómo la tecnología ha ido modelando la demanda de habilidades para el trabajo y generando cambios en los procesos de producción. Plantea que son mayormente requeridas aquellas habilidades que no pueden ser reemplazadas por robots, tales como el pensamiento crítico y habilidades socio-conductuales como la gestión y el reconocimiento de emociones para la mejora del trabajo en equipo. Así mismo, el reporte señala como las tecnologías disruptivas han generado cambios transcendentales en los procesos de producción, ampliando las cadenas de valor global, generando ubicuidad en los puestos de trabajo, así como cambios en la forma de trabajar.

De igual manera, el Foro Mundial de Economía en su informe "El futuro de los empleos, habilidades y estrategia laboral para la cuarta revolución industrial" (World Economic Forum, 2016) plantea que las habilidades sociales, tales como la persuasión, la inteligencia emocional y la enseñanza, entre otros, estarán en mayor demanda para la industria, que las habilidades tecnológicas. El mismo trabajo señala las 10 habilidades que serán requeridas para ejercer algún trabajo en la industria, entre las que se observan el razonamiento matemático, el pensamiento crítico, 
la creatividad, el manejo de personal, la coordinación con otros y la inteligencia emocional.

Así mismo, el Institute for Prospective Technological Studies (IPTS), en el proyecto DIGCOMP (Ferrari, Punie y Brecko, 2013), reconoce que la participación activa en la sociedad 3.0 requiere de un conjunto de competencias relacionadas con las tecnologías digitales, entendidas como "habilidades para la vida", tales como la alfabetización y la aritmética. El proyecto señalado presenta un marco que describe la integración de 21 competencias digitales agrupadas en cinco (5) dimensiones: a) información (identificar, localizar, recuperar, almacenar, organizar y analizar información); b) comunicación (comunicar, compartir, colaborar e interactuar en redes); c) desarrollo de contenidos (desarrollar y editar, integrar y actualizar contenidos multimedia); d) seguridad (proteger su identidad digital, datos, así como el uso seguro de la información); y e) resolución de problemas. Estas competencias digitales son componentes de la ciudadanía digital.

A su vez, Montilva y Montilva (2018) indican la necesidad de buscar mecanismos efectivos de aprendizaje que faciliten la apropiación tecnológica en la sociedad digital. Plantean el pensamiento sistémico y la ontología como dos maneras diferentes de abordar la complejidad de una tecnología. A través de la ontología se puede entender qué es esa tecnología y en qué consiste, y mediante el pensamiento sistémico, se puede concebir y describir a la tecnología como un sistema complejo, facilitando el aprendizaje conceptual necesario para lograr una apropiación tecnológica efectiva.

Lo anterior induce a la necesidad de formar individuos con competencias y habilidades sociales, que además descubran sus propios talentos, aprendan a aprender a lo largo de la vida y a reinventarse (Aragay, 2017). Para ello, la formación universitaria debería orientarse más hacia aspectos humanísticos, que generen innovadoras articulaciones entre el ser, el saber y el hacer, tal y como lo fundamenta la UNESCO (2017) para lograr un desarrollo global sostenible. Una formación multidimensional que apunte a formar individuos capaces de descubrir y construir continuamente el conocimiento, capacitados para crear e innovar y comprometidos con la sociedad a la que pertenecen (Sandia y otros, 2018).

\section{Caracterización de la ciudadanía del nuevo milenio}

Se ha señalado que la ciudadanía global de esta sociedad digital supone la comprensión y uso de tecnologías digitales para el accionar social, cultural, económico y humano, así como la aplicación de conductas y principios que la rigen, que cambian los modos del ejercicio de la ciudadanía tradicional (Rivera Vargas, 2018).

Formar para la sociedad digital implica que los individuos desarrollen ciertas actitudes y competencias que les permitan identificarse y luchar por el bien colectivo, con un conocimiento profundo de los problemas complejos, capaces de enfrentarlos y darles soluciones, con un pensamiento crítico, creativo y sistémico y con 
competencias comunicacionales y habilidades para el trabajo colaborativo. La tabla 1 presenta las dimensiones que agrupan las competencias que deben caracterizar a los ciudadanos y ciudadanas del milenio.

Tabla 1. Dimensiones que describen las competencias de los ciudadanos y ciudadanas del nuevo milenio

\begin{tabular}{|l|l|}
\hline \multicolumn{1}{|c|}{ Dimensión } & \multicolumn{1}{c|}{ Descripción } \\
\hline Ciudadanía & $\begin{array}{l}\text { Capacidad de ser consciente de la importancia del respeto a los } \\
\text { deberes y derechos en la sociedad. }\end{array}$ \\
\hline Creatividad & $\begin{array}{l}\text { Capacidad de generar por sí mismo o en grupo ideas y proyectos } \\
\text { originales para dar solución a problemas globales. }\end{array}$ \\
\hline Colaboración & $\begin{array}{l}\text { Capacidad de trabajar en equipo para alcanzar objetivos } \\
\text { comunes. }\end{array}$ \\
\hline Conocimiento & $\begin{array}{l}\text { Capacidad de comprender, por medio de la razón, la naturaleza, } \\
\text { cualidades y relaciones de las cosas; flexibilidad cognitiva; } \\
\text { razonamiento lógico y matemático. }\end{array}$ \\
\hline Pensamiento Crítico & $\begin{array}{l}\text { Capacidad de analizar y entender situaciones y problemas } \\
\text { complejos y resolverlos de manera exitosa; capacidad de } \\
\text { visualización. }\end{array}$ \\
\hline Comunicación & $\begin{array}{l}\text { Capacidad de transmitir y entender mensajes de manera eficaz a } \\
\text { través de diferentes canales. }\end{array}$ \\
\hline Compromiso & $\begin{array}{l}\text { Capacidad de tomar conciencia de la importancia de cumplir con } \\
\text { las responsabilidades asumidas. }\end{array}$ \\
\hline $\begin{array}{l}\text { Inteligencia } \\
\text { emocional }\end{array}$ & $\begin{array}{l}\text { Actitud positiva, disposición para aprender y enseñar, } \\
\text { perseverancia; capacidad para coordinar, trabajar y negociar con } \\
\text { otros. }\end{array}$ \\
\hline $\begin{array}{l}\text { Pensamiento } \\
\text { sistémico }\end{array}$ & $\begin{array}{l}\text { Capacidad para concebir, describir y analizar sistemas complejos; } \\
\text { para emitir juicio y tomar decisiones. }\end{array}$ \\
\hline $\begin{array}{l}\text { Competencias } \\
\text { digitales }\end{array}$ & $\begin{array}{l}\text { Capacidad de apropiación, manejo y control de tecnologías } \\
\text { digitales. }\end{array}$ \\
\hline
\end{tabular}

Fuente: Elaboración propia.

La incidencia de las tecnologías digitales en la educación y la necesidad de formar los individuos digitales ha llevado, a su vez, a transformar los enfoques educativos. Se observa un rediseño de los espacios de aprendizaje en los que se transforma el aula en un "aula extendida", incorporando las tecnologías en los procesos de formación, ampliando las posibilidades de complementar el aprendizaje con el desarrollo de actividades a través de estos medios, posibilitando el aprendizaje activo.

Aparecen conceptos como el "aula invertida" (flipped classroom), donde el estudiante ocupa el tiempo en el aula (la presencialidad) resolviendo tareas, analizando problemas, trabajando en equipo, mientras el docente destina este tiempo 
para aclarar dudas complejas a través de la reflexión o la resolución de problemas. El tiempo fuera del aula (la virtualidad) es para investigar, revisar, estudiar y repasar el material que normalmente es impartido en clases magistrales.

En este mismo orden de ideas, términos como las aulas inteligentes (smartrooms) en los que los dispositivos de comunicación remota y las conferencias web, entre otros, se integran e interconectan para facilitar el desarrollo de proyectos basados en la colaboración, la movilidad y la flexibilidad.

Esta integración de espacios de aprendizaje presenciales (físicos) y virtuales ha llevado a generar nuevas formas de aprendizaje híbrido o mixto (blended learning). Surgen conceptos como el aprendizaje "poli-sincrónico", en el que el uso de una mezcla de canales de comunicación a distancia, presencial, asíncrona y síncrona, para la participación de los estudiantes desde diversos lugares, es clave para el proceso educativo.

Conceptos como el "aprendizaje profundo", que promueve la formación en el pensamiento crítico, la resolución de problemas, el aprendizaje autónomo y colaborativo, han generado enfoques educativos que fomentan experiencias de aprendizaje más activas y que permiten la construcción del conocimiento significativo, tales como el "aprendizaje basado en proyectos". Así encontramos el término "makerspaces", que identifica a ambientes físicos que han emergido como entornos prometedores de aprendizaje experiencial que apoyan el desarrollo de habilidades como la colaboración, el pensamiento crítico, la creatividad y la innovación.

En la Universidad de Los Andes, en Venezuela, desde el año 2009, se ha desarrollado y utilizado una estrategia educativa denominada RAIS (Reproducción del Ambiente de Innovación en el Salón de clase), que plantea la transformación de la formación, centrada en el manejo integral del conocimiento para crear, hacer, innovar y producir, a través del desarrollo de un producto. Esta estrategia apunta a formar individuos con actitudes y competencias relacionadas con la innovación y el emprendimiento y con valores de seres universales, características señaladas para la ciudadanía del nuevo milenio (Sandia y otros, 2018).

Por otro lado, el aprendizaje-servicio es un concepto educativo orientado hacia la responsabilidad y transformación social, con un enfoque innovador y de carácter experiencial que integra el aprendizaje académico con la reflexión crítica y el servicio a la comunidad, permitiendo el crecimiento personal y la responsabilidad cívica, con el objetivo de formar individuos generadores del cambio social (Aramburuzabala, Cerrillo y Tello, 2015).

\section{El aprendizaje-servicio como mecanismo para la formación ciudadana del nuevo milenio}

El servicio comunitario, la educación ciudadana y ambiental, el aprendizaje experiencial basado en el servicio a la comunidad, así como el aprendizaje-servicio, son cada vez más evidentes en las instituciones de educación superior. 
El aprendizaje basado en la comunidad o aprendizaje-servicio es un enfoque educativo que combina el servicio a la comunidad con el aprendizaje académico para proporcionar una formación pragmática y gradual y a la vez cubrir las necesidades de la sociedad. Este enfoque permite a los estudiantes adquirir, practicar y aplicar los conocimientos y habilidades propias de la asignatura que cursan y, al mismo tiempo, desarrollar competencias y actitudes de una ciudadanía efectiva al identificar y actuar sobre problemas reales que afectan a sus propias comunidades, lo que conlleva a que el aprendizaje tenga un propósito y significado (Melaville, Berg y Blank, 2006).

Este enfoque educativo, además de proporcionar oportunidades para desarrollar servicios que cubren las necesidades de una comunidad, permite a los estudiantes adquirir habilidades relacionadas con la comunicación y las relaciones interpersonales, la colaboración, el trabajo en equipo, y la resolución de problemas, habilidades necesarias para la ciudadanía del milenio (Arantes do Amaral y Lino dos Santos, 2018).

El aprendizaje-servicio es, además, una estrategia especialmente adecuada para formar ciudadanas y ciudadanos activos e informados, ya que proporciona oportunidades para conectar el currículo con la comunidad y desarrollar habilidades y actitudes de participación ciudadana; así como valores y conocimientos inherentes a la democracia participativa, mientras trabajan en la resolución del problema comunitario (Wade, 2000).

Según Lucas y Arantzazu (2012), este tipo de estrategia le proporciona al estudiante la posibilidad de participar en la resolución de problemas complejos y reales que afectan a la comunidad, permitiendo oportunidades de aprendizaje constructivas, reales y significativas, aumentando la motivación y favoreciendo el desarrollo social, emocional y profesional.

Portillo (2018) en su trabajo "El aprendizaje-servicio, la educación para el desarrollo sostenible y las competencias transversales, una vía hacia sociedades sostenibles" determina que efectivamente el aprendizaje-servicio está conectado con la capacidad de generar conciencia crítica y con el desarrollo sostenible.

Puede observarse, tal y como lo señalan Lucas y Arantzazu (2012), que la estrategia aprendizaje-servicio contribuye al desarrollo de las distintas competencias genéricas señaladas como características para la ciudadanía del milenio. A través del aprendizaje-servicio se fomenta competencias relacionadas con la ciudadanía global, el compromiso, la creatividad, la comunicación, las relaciones interpersonales y el trabajo en equipo, así como el pensamiento crítico y sistémico.

\section{TECNOLOGÍAS DIGITALES DE APOYO AL APRENDIZAJE-SERVICIO VIRTUAL}

García, Ruiz y Del Pozo (2016) señalan como aprendizaje-servicio virtual a la modalidad virtual o en línea de esta estrategia educativa, que está caracterizada por el nivel de mediación tecnológica que se requiere en cada proceso educativo. 
Esta mediación tecnológica varía dependiendo de qué tecnologías se utilicen y cómo ellas se integran a los procesos de enseñanza y aprendizaje. Los espacios virtuales de aprendizaje (EVA) constituyen la forma de mediación tecnológica más avanzada, dado que integran en un solo ambiente un conjunto de tecnologías apropiadas para las actividades educativas que se pretendan realizar. Alves, Miranda y Morais (2016) definen a un EVA como "un espacio virtual que integra un conjunto de recursos y herramientas digitales que favorecen la interacción entre estudiantes, docentes y contenidos, con potencialidades para la implementación de estrategias pedagógicas de aprendizaje".

Siendo el aprendizaje-servicio una modalidad de aprendizaje relativamente reciente, se hace necesario identificar los elementos fundamentales que componen un espacio virtual para el aprendizaje-servicio (EVApS), así como establecer su relación con las tecnologías digitales a través de un instrumento que las unifique y cree el entorno necesario para que los procesos de enseñanza, aprendizaje y servicios se den con el apoyo de tales tecnologías. Para ello, hemos elaborado un modelo que identifica los aspectos fundamentales que caracterizan a los espacios virtuales para el aprendizaje-servicio y establece las diferencias con otros tipos de EVA. Para la construcción de este modelo nos hemos apoyado en el modelado de sistemas y las arquitecturas de software, dos importantes áreas de la Computación que facilitan la concepción, caracterización y representación formal de sistemas tecnológicos complejos.

\section{Espacios Virtuales para el Aprendizaje-Servicio}

Un espacio virtual es un concepto u objeto ideal creado por el ser humano para hacer referencia a un ambiente en el cual una comunidad o conjunto de personas interactúan a través de una red digital (p.ej., Internet, Intranet o una red de área local). Este objeto ideal tiene, por lo general, un propósito definido o predeterminado.

Internet y, concretamente, la Web han ofrecido la oportunidad de crear nuevos espacios de enseñanza-aprendizaje alternos al aula de clase presencial. Estos ciberespacios, denominados espacios virtuales de aprendizaje (EVA), son ambientes de interacción, alojados en redes de datos, sin limitaciones espacio-temporales en su utilización y orientados a apoyar procesos de enseñanza y aprendizaje. Los EVA integran: (1) un amplio conjunto de tecnologías digitales que se acceden a través de interfaces creadas con la tecnología WWW; (2) nuevas metodologías, técnicas y estrategias de enseñanza y aprendizaje que aprovechan las oportunidades que ofrece el ciberespacio; y (3) nuevos procesos asociados a la gestión académica, a la enseñanza, al aprendizaje, así como al uso y gestión de las tecnologías empleadas en los EVA.

Visto como un sistema, un EVApS tiene cinco aspectos fundamentales que lo caracterizan como tal: una intencionalidad, un ambiente, una estructura, una 
funcionalidad y una dinámica o comportamiento. Cada uno de estos aspectos se describen seguidamente.

\section{Intencionalidad de un EVApS}

Un EVApS es un sistema intencional que se diseña, construye y utiliza para lograr objetivos preestablecidos relacionados con la prestación de servicios a comunidades $\mathrm{u}$ organizaciones sociales, en conjunto con objetivos de aprendizaje propios de su función educativa. Su intencionalidad es, por consiguiente, dual; por un lado, persigue objetivos de aprendizaje asociados al dominio o área de conocimiento en la cual se enmarca el EVApS y, por otro, busca alcanzar los objetivos propios del aprendizaje-servicio hacia el cual se orienta.

Un EVApS es un tipo especial de sistema de aprendizaje virtual que está dirigido a apoyar la formación de ciudadanos y ciudadanas para este nuevo siglo y para un mundo global e interconectado que sean capaces, como ya lo mencionamos anteriormente, de enfrentar la incertidumbre, desarrollar un pensamiento crítico, innovar, generar nuevo conocimiento, dominar las tecnologías emergentes y usarlas para el bien común. La formación de este individuo se sustenta en el desarrollo de un conjunto de competencias específicas tales como las descritas en la tabla 1.

\section{Estructura y ambiente de un EVApS}

Estructuralmente, un EVA está compuesto de partes que se integran para ofrecer una funcionalidad que emerge de las relaciones entre estas y que solo es posible cuando están interconectadas y operando. Estos componentes interactúan con el propósito de alcanzar un conjunto de objetivos de aprendizaje preestablecidos y asociados al aprendizaje-servicio para el cual el EVApS ha sido diseñado y desarrollado. La figura 1 identifica y relaciona estos componentes y establece el contexto, ambiente $o$ entorno social en cual un EVApS existe y con el cual interactúa.

Como sistema abierto que es, un EVAPpS interactúa con su entorno social formado por la institución académica, dentro de la cual el EVApS se ubica y a la que sirve como medio de educación para la ciudadanía; así como por una o más comunidades, organizaciones o grupos de individuos que se benefician de las acciones del aprendizaje-servicio que le dan sentido y pertinencia al EVApS. 
Figura 1. Componentes de un EVApS, sus relaciones y su entorno social

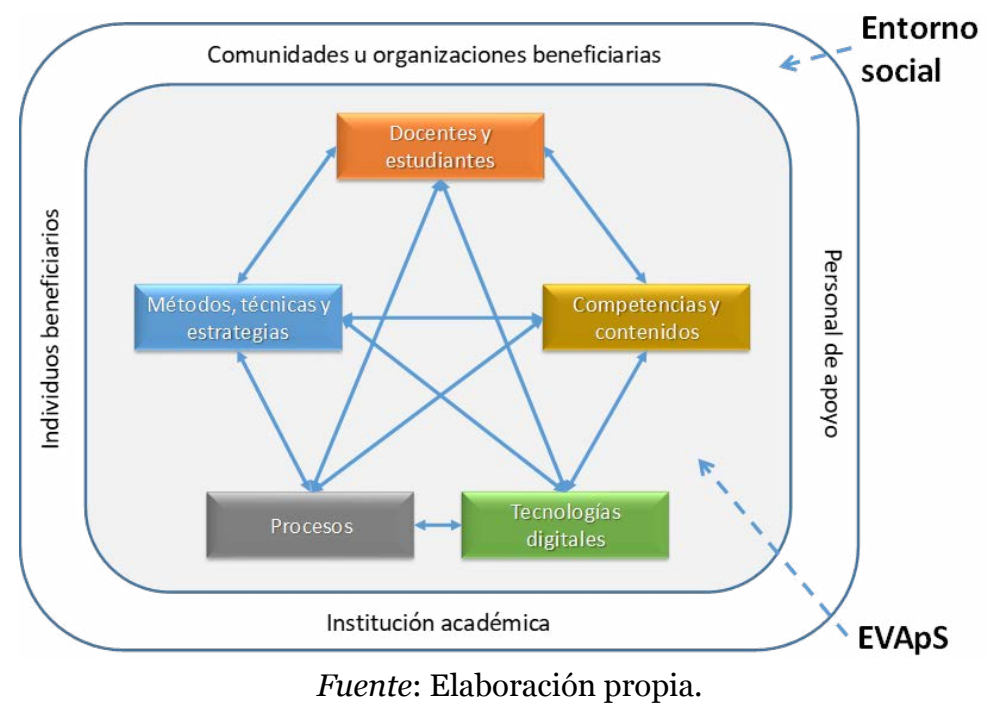

El componente humano de este sistema está integrado por docentes, estudiantes, personal académico-administrativo, que apoya a los docentes en la función educativa, y personal técnico que da soporte a los docentes y estudiantes en el uso de las tecnologías digitales que conforman el EVApS.

Los objetivos de aprendizaje del sistema se traducen en un conjunto de competencias orientadas al aprendizaje-servicio (ver tabla 1). Estrechamente relacionados con estas competencias están los contenidos; esto es, la información necesaria para que el estudiante desarrolle las competencias cognitivas que le permitirán llevar a cabo la prestación de servicios en un determinado sector, comunidad o dominio social.

El desarrollo de estas competencias, por parte de los estudiantes, depende en buena medida de los métodos, técnicas, prácticas y estrategias de enseñanzaaprendizaje que los docentes utilicen. El aprendizaje-servicio representa el componente metodológico central de todo EVApS. Este componente integra un conjunto de métodos, prácticas, estrategias y técnicas orientadas a lograr "una participación auténtica del alumnado en la comunidad, una participación orientada al logro del bien común y a la adquisición de valores y virtudes cívicas" (Puig Rovira, Gijón Casares, Martín García y Rubio Serrano, 2011). Por lo general, la metodología del aprendizaje-servicio se asocia y se utiliza en combinación con otras, tales como: el aprendizaje colaborativo, el aprendizaje por descubrimiento, el aprendizaje activo y el aprendizaje basado en proyectos (Sandia, 2018). Este último en particular resulta fundamental para definir, organizar, planificar y controlar las actividades asociadas a la prestación de servicios comunitarios. 
El componente metodológico de un EVApS está estrechamente vinculado a los procesos de enseñanza, aprendizaje y servicio que tienen lugar durante la puesta en ejecución del sistema. Cada uno de estos procesos está compuesto por un conjunto interrelacionado de actividades que deben ser ejecutadas por los actores pertinentes al proceso y cuyos resultados han de contribuir a la consecución de los objetivos del sistema. Las actividades que integran los procesos de enseñanza y aprendizaje dependen de los métodos, prácticas, estrategias y técnicas que fueron seleccionadas por el docente; mientras que las actividades de prestación de servicios comunitarios son propias del tipo de servicio hacia el cual se dirige el aprendizaje.

El componente tecnológico actúa como el medio a través del cual se ejecutan y/o se le da apoyo a los procesos de enseñanza, aprendizaje y servicio. La figura 2 identifica un amplio conjunto de tecnologías aplicables en la construcción de un EVApS. Estas tecnologías se integran mediante la Web e interactúan a través de redes digitales para crear así un espacio virtual de aprendizaje.

\section{Funcionalidad y comportamiento de un EVApS}

Un EVApS se construye o desarrolla mediante la integración de un conjunto de tecnologías digitales que se acceden a través de la Web y se comunican a través de redes digitales, tales como Internet, Intranet o redes de alcance local. La figura 2 muestra la arquitectura general de un EVApS expresada en términos de capas independientes que se comunican entre sí, para proporcionar un conjunto de funciones disponibles a los usuarios del EVApS.

Figura 2. Arquitectura general de un EVApS

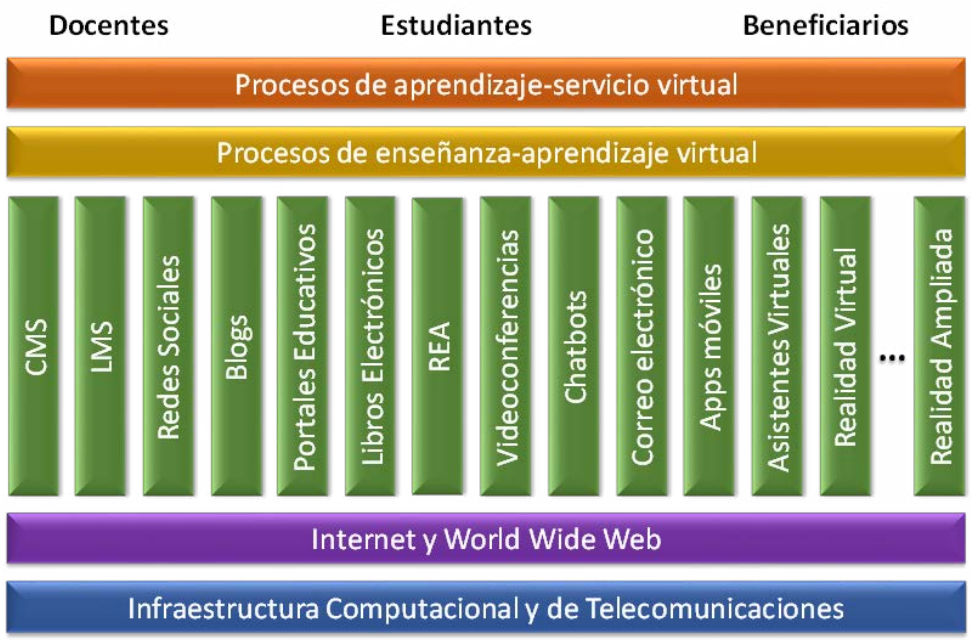

Fuente: Elaboración propia. 
Cada capa arquitectónica actúa como una caja negra que presta un conjunto de funciones o servicios a las capas superiores, sin que estas últimas requieran conocer como estas funciones o servicios están implementados. A esta característica arquitectónica se le conoce como propiedad de encapsulamiento y es fundamental para la comprensión de una tecnología digital. Así, por ejemplo, la capa compuesta por el Internet y la WWW (World Wide Web) provee los servicios necesarios para desarrollar, utilizar y mantener la capa de tecnologías digitales para educación; la cual, a su vez, provee a los usuarios las funciones necesarias para ejecutar los procesos virtuales de enseñanza, aprendizaje y servicio.

Esta arquitectura organiza los componentes de un EVApS en tres grupos, a saber, componentes tecnológicos, componentes de procesos y componente humano.

Los componentes tecnológicos, a su vez, se dividen en componentes de base y componentes tecnológicos educativos. La base de la arquitectura está formada por un conjunto de servidores locales o en la Nube y dispositivos de telecomunicaciones que facilitan el acceso remoto a los servicios de Internet y WWW.

En el centro de esta arquitectura se ubica un conjunto de tecnologías digitales orientadas a apoyar los procesos educativos, integradas a través de la WWW. Este conjunto varía de un EVApS a otro, dependiendo de las necesidades de cada curso o asignatura. Los sistemas de gestión de contenidos CMS (p.ej., WordPress, Joomla y Blogger) y los sistemas de gestión de aprendizaje LMS (p.ej., MOODLE, LearnPress, Blackboard, etc.) constituyen las herramientas tecnológicas más utilizadas para la construcción de espacios virtuales de aprendizaje.

Las capas superiores proveen los servicios o funcionalidad necesaria para que los actores del aprendizaje-servicio lleven a cabo sus actividades de enseñanza, aprendizaje y servicio. Por ejemplo, los estudiantes pueden:

- Acceder a los contenidos del curso o asignatura.

- Interactuar con el docente, con sus compañeros o con los beneficiarios del servicio, bien a tiempo real (vía chat, videoconferencia o llamadas telefónicas) o a tiempo diferido (vía correo electrónico, mensajería de texto o redes sociales).

- Utilizar una amplia variedad de recursos educativos abiertos (REA) disponibles en repositorios especializados o de libre acceso, tales como: artículos, libros electrónicos, revistas, videos, etc.

- Realizar actividades prácticas o de laboratorio haciendo uso de la realidad virtual o ampliada.

- Medir su progreso en el curso y realizar las evaluaciones requeridas.

Esta manera de concebir a un EVApS le da al docente la posibilidad de crear un espacio virtual adaptado a las necesidades particulares de cada curso. Mediante la selección e integración de las tecnologías digitales que requiere para lograr el desarrollo de las competencias propuestas en su asignatura, el docente podrá 
crear un espacio virtual más particularizado y ajustado a los requerimientos de los objetivos planteados en su asignatura.

\section{Aplicabilidad de las tecnologías digitales en EVApS}

La aplicabilidad de una tecnología depende de la utilidad que ella tenga para apoyar los procesos del entorno donde ella se utilice. Montilva (2018) describe, por ejemplo, diversas maneras de aplicar tecnologías digitales en el contexto de la educación superior, que van desde los procesos de enseñanza y aprendizaje hasta los procesos administrativos.

En el diseño y desarrollo de un EVApS puede aplicarse, tal como se destaca en la arquitectura descrita en la sección anterior, un número considerable de tecnologías digitales orientadas a apoyar los procesos virtuales de enseñanza, aprendizaje y servicio.

En función de la utilidad que las tecnologías digitales tienen para apoyar el aprendizaje-servicio, hemos clasificado estas tecnologías en cinco categorías no exhaustivas y no disyuntivas (ver tabla 2). Cada una de ellas se describe a continuación.

\section{Tecnologías colaborativas}

Bajo esta categoría se agrupan todas aquellas tecnologías que contribuyen, de diversas maneras, a lograr una interacción remota entre docentes y estudiantes, a facilitar el trabajo en equipo y a permitir la colaboración a distancia entre diferentes actores. Las redes sociales y las aplicaciones móviles interactivas constituyen actualmente las tecnologías colaborativas de mayor uso en diferentes ámbitos incluyendo a la educación.

\section{Tecnologías de infraestructura computacional y de comunicaciones}

Tal como lo indica la arquitectura EVApS de la figura 2, la base o plataforma fundamental e indispensable de todo espacio virtual de aprendizaje es la infraestructura computacional y de comunicaciones. Esta infraestructura incluye un conjunto de equipos de cómputo y dispositivos de comunicaciones distribuidos e interconectados a través de Internet $\mathrm{u}$ otra red digital. La Nube representa, en la actualidad, la plataforma computacional de mayor crecimiento y auge, debido a su capacidad para reemplazar los costosos centros de datos presentes aún en muchas universidades iberoamericanas. 


\section{Tecnologías para la gestión de datos, información, conocimientos y procesos}

El manejo de datos, información y/o conocimientos constituye la actividad fundamental de toda tecnología digital. La captura, procesamiento y almacenamiento de datos y su transformación de datos en información; así como la representación digital, almacenamiento, intercambio y aplicación de conocimientos, son funciones típicas de este tipo de tecnologías. A ellas se une el modelado y automatización de procesos, a través de tecnologías tales como: la gestión de procesos de negocio BPM (Business Process Management) y la gestión de proyectos. Todas ellas encuentran usos diferentes para el apoyo de los procesos educativos y de servicios.

\section{Tecnologías inteligentes}

Los avances en Inteligencia Artificial y el carácter ubicuo que han adquirido sus tecnologías en los últimos años abren un abanico de oportunidades para la aplicación de ellas, tanto en los procesos educativos como en los de servicios. Existe un amplio número de aplicaciones inteligentes que pueden utilizarse para apoyar el desarrollo de competencias, tales como la gestión de información y conocimiento y la solución de problemas. Los robots educativos son, además, recursos que están ganando gradualmente un espacio privilegiado en ambientes educativos.

\section{Tecnologías visuales}

La realidad virtual, la realidad ampliada y los videojuegos tienen, cada vez más, una amplia aplicabilidad en la educación. La realidad virtual y la ampliada, por ejemplo, son herramientas excelentes para simular experimentos en laboratorios virtuales; así como para la simulación de escenarios y eventos históricos. Por otro lado, los videojuegos han sido tradicionalmente empleados, de muy diversas maneras, para estimular la creatividad del estudiante.

Cada una de las tecnologías incluidas en la tabla 2 se ha asociado a una o más competencias para la ciudadanía del mileno; ello con la finalidad de reflejar un posible uso en actividades educativas o de servicio que contribuyan al desarrollo de dichas competencias. Otras tecnologías pueden, por supuesto, considerarse durante el proceso de diseño y desarrollo de un EVApS.

La lista presentada en la tabla 2 es de carácter ilustrativo. Su intención es mostrar el potencial que las tecnologías digitales tienen para apoyar el desarrollo en el estudiante de las principales competencias del ciudadano y ciudadana del milenio. Qué tecnologías digitales integrar a un EVApS depende del propósito del curso. Zandy y Franco (2014), por ejemplo, integran mapas digitales a un EVApS con la finalidad de darle a sus estudiantes un sentido virtual del espacio geográfico donde el aprendizaje-servicio, realizado a distancia, se lleva a cabo. 
Tabla 2. Aplicabilidad de las tecnologías digitales en el desarrollo de las competencias del aprendizaje-servicio

\begin{tabular}{|c|c|c|c|c|c|c|c|c|c|c|}
\hline & & \multicolumn{9}{|c|}{ Competencias para aprendizaje-servicio } \\
\hline \multicolumn{2}{|r|}{ Tecnologías digitales } & 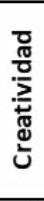 & $\begin{array}{l}\frac{0}{0} \\
\frac{\pi}{\pi} \\
\frac{0}{0} \\
\frac{\pi}{0} \\
0\end{array}$ & 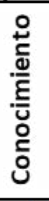 & 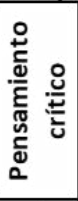 & 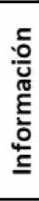 & 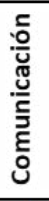 & 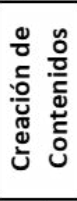 & 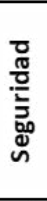 & 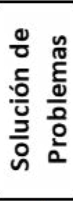 \\
\hline \multirow{5}{*}{ I } & Aplicaciones móviles & $\sqrt{ }$ & $\sqrt{ }$ & $\sqrt{ }$ & $\sqrt{ }$ & $\sqrt{ }$ & $\sqrt{ }$ & $\sqrt{ }$ & $\sqrt{ }$ & $\sqrt{ }$ \\
\hline & Redes sociales & $\sqrt{ }$ & $\sqrt{ }$ & & & $\sqrt{ }$ & $\sqrt{ }$ & $\sqrt{ }$ & & $\sqrt{ }$ \\
\hline & Videoconferencia & & $\sqrt{ }$ & & & & $\sqrt{ }$ & & & \\
\hline & Chat y mensajería instantánea (IM) & & $\sqrt{ }$ & & & & $\sqrt{ }$ & & & \\
\hline & Correo electrónico & & $\sqrt{ }$ & & & $\sqrt{ }$ & $\sqrt{ }$ & & & \\
\hline \multirow{5}{*}{ II } & La Nube & & $\sqrt{ }$ & & & $\sqrt{ }$ & $\sqrt{ }$ & & $\sqrt{ }$ & \\
\hline & $5 G$ & & & & & & $\sqrt{ }$ & & & \\
\hline & Redes híbridas & & & & & & $\sqrt{ }$ & & & \\
\hline & Internet de las Cosas (IoT) & & & & & & $\sqrt{ }$ & & & \\
\hline & Telefonía inteligente & & $\sqrt{ }$ & & & $\sqrt{ }$ & $\sqrt{ }$ & & & \\
\hline \multirow{11}{*}{ III } & Big Data & & & & & $\sqrt{ }$ & & & & \\
\hline & Analítica de Datos & & & $\sqrt{ }$ & $\sqrt{1}$ & $\sqrt{ }$ & & & & $\sqrt{ }$ \\
\hline & Blockchain & & & & & $\sqrt{ }$ & & & $\sqrt{ }$ & \\
\hline & Asistentes virtuales & & & $\sqrt{ }$ & & $\sqrt{ }$ & $\sqrt{ }$ & & & \\
\hline & Gestión de Contenidos (CMS) & & $\sqrt{ }$ & & & $\sqrt{ }$ & $\sqrt{ }$ & $\sqrt{ }$ & & \\
\hline & Gestión de Aprendizaje (LMS) & & $\sqrt{ }$ & & & $\sqrt{ }$ & $\sqrt{ }$ & $\sqrt{ }$ & & \\
\hline & Editores de contenidos & & & & & $\sqrt{ }$ & & $\sqrt{ }$ & & \\
\hline & Portales educativos & & $\sqrt{ }$ & & & $\sqrt{ }$ & $\sqrt{ }$ & $\sqrt{ }$ & & \\
\hline & Gestión de conocimiento (KMS) & & & $\sqrt{ }$ & $\sqrt{ }$ & $\sqrt{ }$ & & $\sqrt{ }$ & & $\sqrt{ }$ \\
\hline & Gestión de procesos y proyectos (BPM) & & $\sqrt{ }$ & & & & $\sqrt{ }$ & & & $\sqrt{ }$ \\
\hline & Recursos Educativos Abiertos (REA) & $\sqrt{ }$ & $\sqrt{ }$ & $\sqrt{ }$ & $\sqrt{ }$ & $\sqrt{ }$ & $\sqrt{ }$ & $\sqrt{ }$ & $\sqrt{ }$ & $\sqrt{ }$ \\
\hline \multirow{6}{*}{ IV } & Agentes inteligentes & & & $\sqrt{ }$ & & $\sqrt{ }$ & & & & $\sqrt{ }$ \\
\hline & Aprendizaje de máquina & & & $\sqrt{ }$ & & $\sqrt{ }$ & & & & $\sqrt{ }$ \\
\hline & Aprendizaje profundo & & & $\sqrt{ }$ & & $\sqrt{ }$ & & & & $\sqrt{ }$ \\
\hline & Sistemas biométricos & & & & & & & & $\sqrt{ }$ & \\
\hline & Robots educativos & $\sqrt{ }$ & & $\sqrt{ }$ & & $\sqrt{ }$ & $\sqrt{ }$ & & & \\
\hline & Traductores & & & & & $\sqrt{ }$ & $\sqrt{ }$ & & & \\
\hline \multirow{3}{*}{ v } & Realidad virtual & $\sqrt{ }$ & & & & & & $\sqrt{ }$ & & \\
\hline & Realidad ampliada & $\sqrt{ }$ & & & & & & $\sqrt{ }$ & & \\
\hline & Videojuegos & $\sqrt{ }$ & & & & & & & & \\
\hline
\end{tabular}

Fuente: Elaboración propia.

\section{CONCLUSIONES}

La sociedad digital en la que nos encontramos inmersos, caracterizada por la conectividad entre personas, información y conocimiento, así como la necesidad de la sostenibilidad y la reciprocidad interdependiente, nos obliga a que la formación, la educación, en todos los niveles, se transforme y se adapte a esta realidad. Esto 
supone un rebote trascendental en el alcance y la complejidad de la inteligencia colectiva (Schwab, 2016), formando ciudadanos y ciudadanas del mundo para un nuevo milenio, con actitudes, habilidades y destrezas orientadas, principalmente, a reconocerse como seres humanos universales, cuyo accionar se fundamenta en la libertad, la autonomía y la responsabilidad; seres capaces de aprender a enfrentar la incertidumbre y desarrollar un pensamiento crítico, dispuestos para innovar y generar nuevo conocimiento.

Formar para la sociedad digital requiere que los individuos desarrollen ciertas actitudes y competencias que les permitan identificarse y luchar por el bien colectivo, con un conocimiento profundo de los problemas complejos, capaces de enfrentarlos y darles soluciones, con un pensamiento crítico, creativo y sistémico y con competencias comunicacionales y habilidades para el trabajo colaborativo. Pero además debe suponer la comprensión y uso de tecnologías digitales, para el accionar social, cultural, económico y humano, así como la aplicación de conductas y principios que la rigen, que cambian los modos del ejercicio de la ciudadanía tradicional.

El enfoque educativo del aprendizaje-servicio permite formar ciudadanas y ciudadanos activos e informados, proporciona oportunidades para conectar el currículo con la comunidad y desarrollar habilidades y actitudes de participación ciudadana, así como valores y conocimientos inherentes a la democracia participativa, mientras trabajan en la resolución de problemas complejos reales en la comunidad; este enfoque, además, permite que los estudiantes adquieran habilidades relacionadas con la comunicación y las relaciones interpersonales, la colaboración, el trabajo en equipo y la resolución de problemas, y está conectado con la capacidad de generar conciencia crítica y con el desarrollo sostenible.

Es importante resaltar que el definir y establecer el conjunto de competencias que caracterizan la ciudadanía del nuevo milenio permite determinar, con mayor precisión, los objetivos de aprendizaje para los proyectos de aprendizaje-servicio.

Por otro lado, la incorporación de las tecnologías digitales, específicamente a través de un entorno virtual para el aprendizaje-servicio (EVApS), supone un elemento innovador que potencia el desarrollo de las competencias requeridas para la ciudadanía del milenio.

Concebir un EVApS como un sistema en el que se puede seleccionar e integrar distintas tecnologías digitales, para lograr el desarrollo de las competencias propuestas en su asignatura, da la posibilidad al docente de crear un espacio virtual más particularizado y ajustado a los requerimientos de los objetivos planteados y del alcance del aprendizaje-servicio. De esta manera, los EVApS se consolidan como medios tecnológicos fundamentales para promover la construcción de espacios de participación y compromiso social a través de la ejecución de proyectos y acciones comunitarias, sustentados en tecnologías digitales que le dan una mayor expansión y alcance, promoviendo además el desarrollo de las competencias señaladas como requeridas para la ciudadanía del nuevo milenio. 


\section{REFERENCIAS}

Alves, P., Miranda, L., y Morais, C. (2016). The Importance of Virtual Learning Environments in Higher Education. En D. Fonseca y E. Redondo, (Eds.), Handbook of Research on Applied E-Learning in Engineering and Architecture Education (404-425). Hershey, PA: IGI Global.

Alves, P., Miranda, L., y Morais, C. (2017). The Influence of Virtual Learning Environments in Students' Performance. Universal Journal of Educational Research 5(3), 517-527. doi: 10.13189/ ujer.2017.050325.

Aragay Tusell, X. (2017). Entrevista Reimaginando la Educación. Recuperado de https://observatorio.itesm.mx/ edu-news/2017/11/6/entrevista-xavieraragay-reimaginando-la-educacion

Aramburuzabala, P., Cerrillo, R., y Tello, I. (2015). Aprendizaje-servicio: Una propuesta metodológica para la introducción dela sostenibilidad curricular en la Universidad. Universidad Autónoma de Madrid. Revista Profesorado, 19(1). Recuperado de https://www.ugr. es/ recfpro/rev191ART5.pdf

Arantes do Amaral, J. A., y Lino dos Santos, R. J. R. (2018). Combining Project-Based Learning and Community-Based Research in a Research Methodology Course: The Lessons Learned. International Journal of Instruction, 11(1), 47-60. doi: https:// doi.org/10.12973/iji.2018.1114a

Bakhshi, H., Downing, J., Osborne, M., y Schneider, P. (2017). The Future of Skills: Employment in 2030. London: Pearson and Nesta. Recuperado de https:// futureskills.pearson.com/

Ferrari, A., Punie, Y., y Brecko, B. (2013). DIGCOMP: A Framework for Developing and Understanding Digital Competence in Europe. Recuperado de https:// ec.europa.eu/jrc/en/publication/ eur-scientific-and-technical-researchreports/digcomp-framework-developing- and-understanding-digital-competenceeurope

García-Gutiérrez, J., Ruiz-Corbella, M., y Del Pozo Armentia, A. (2016). Cuando la comunidad se expande: ciudadanía global y aprendizaje-servicio virtual (apsv). Educación y Diversidad, 10(2), 63-75. Recuperadodehttps://www.researchgate. net/publication/321383105 CUANDO LA COMUNIDAD SE EXPANDE CIUDADANIA GLOBAL Y APRENDIZAJE-SERVICIO VIRTUAL

Herrera Mosquera, L. (2017). Impact of Implementing a Virtual Learning Environment (VLE) in the EFL Classroom. Ikala. Revista de Lenguaje y Cultura, 22(3), 479-498. doi: 10.17533/udea.ikala. v22no3ao7.

Kurt, E., y Tingoy, O. (2017). The acceptance and use of a virtual learning environment in higher education: an empirical study in Turkey, and the UK. International Journal of Educational Technology in Higher Education, 14-26. doi: 10.1186/ s41239-017-0064-z.

Lucas Mangas, S., y Arantzazu Martínez, O. (2012). La implantación y difusión del Aprendizaje-Servicio en el contexto educativo español. Retos de futuro de una metodología de enseñanza-aprendizaje para promover la innovación en la Educación Superior. Recuperado de https://www.cidui.org/revistacidui/ index.php/cidui/article/view/122

Melaville, A., Berg, A., y Blank, M. (2006). Community-Based Learning: Engaging Students for Success and Citizenship. Coalition for Community Schools. Recuperado de https://eric. ed.gov/?id=ED491639

Montilva, J. (2018). Tecnologías Digitales en la Educación Superior. Mérida, Venezuela, Blog de la Academia de Mérida. Recuperado de https:// blogacademiademerida.org.ve/ 
tecnologias-digitales-en-la-educacionsuperior/

Montilva, J., y Montilva, W. (2018). Un método ontológico-sistémico para el aprendizaje conceptual de tecnologías digitales. Revista Ciencia e Ingeniería, 39(3), 269-278. ISSN 1316-7081. ISSN. Elect. 2244-8780.

Morin, E. (2002). Los siete saberes necesarios para la educación del futuro. UNESCO. Recuperado de http://unesdoc.unesco. org/images/0011/001177/117740so.pdf

Portillo, N. (2018). El aprendizaje servicio, la educación para el desarrollo sostenibleylas competencias transversales, una vía hacia sociedades sostenibles. En V. Martínez y otros (editores): El aprendizaje servicio en la universidad. Una metodología docente y de investigación al servicio de la justicia social y el desarrollo sostenible. (120-125). Comunicación Social Ediciones y Publicaciones, Salamanca. Recuperado de https://rio.upo.es/xmlui/ bitstream/handle/10433/6323/El aprendizaje-servicio en la universidad. pdf? sequence $=1$ \&isAllowed $=\mathrm{y}$

Puig Rovira, J. M., Gijón Casares, M., Martín García, X., y Rubio Serrano, L. (2011). Aprendizaje-Servicio y Educación para la Ciudadanía. Revista de Educación, número extraordinario. 45-67. Recuperado de http://www. revistaeducacion.mec.es/re2011 03.htm

Rivera Vargas, P. (2018). Sociedad digital y ciudadanía: un nuevo marco de análisis. Recuperado de https://www.researchgate.net/ publication/326710857 SOCIEDAD DIGITAL Y CIUDADANIA UN NUEVO MARCO DE ANALISIS

Sandia Saldivia, B. (2018). Tecnologías y Transformación en los Enfoques Educativos. Mérida, Venezuela. Blog de la Academia de Mérida. Recuperado de https://blogacademiademerida.org.ve/ tecnologias-y-transformacion-en-losenfoques-educativos/
Sandia Saldivia, B., Arnal Sandia, G., Moreno, Y., Mujica, A., Hernández, D., y Páez Monzón, G. (2018). Simulando el Ambiente de Innovación en el Salón de Clase. Aprendizaje Basado en Productos. VISUS Revista Politécnica de Desarrollo e Innovación, 2(1), 10-20.

Serrano Zaneti, M. (2014). Software Engineering: A Complex Systems Approach. Zurich: Südwestdeutscher Verlag für Hochschulschriften.

Schwab, K. (2016). The Fourth Industrial Revolution: what it means, how to respond. Recuperado de https://www. weforum.org/agenda/2016/01/thefourth-industrial-revolution-what-itmeans-and-how-to-respond

UNESCO. (2016). Educación para la Ciudadanía Mundial. Preparar a los educandos para los retos del siglo XXI. Recuperado de http://unesdoc.unesco. org/images/0024/002449/244957s.pdf

UNESCO. (2017). Education for Sustainable Development Goals Learning Objectives. The Global Education 2030 Agenda. Recuperado de http://unesdoc.unesco. org/images/0024/002474/247444e.pdf

Van den Hoff, R. (2013). Mastering the Global Transition on Our Way to Society 3.o. Society 3.0 Foundation. Recuperado de https://society3o.com/publications/

Wade, R., (Ed.) (2000). Building Bridges: Connecting Classroom and Community through Service-Learning in Social Studies. NCSS Bulletin 97. Recuperado de https://eric.ed.gov/?id=ED446008

World Bank. (2019). World Development Report 2019: The Changing Nature of Work. Washington, DC: World Bank. Recuperado de http://www.worldbank. org/en/publication/wdr2019

World Economic Forum. (2016). The Future of Jobs Employment, Skills and Workforce Strategy for the Fourth Industrial Revolution. Recuperado de http://www3.weforum.org/docs/WEF Future of Jobs.pdf 
Zandy, M. G., y Franco, Z. E. (2014). Grounding Service-Learning in the Digital Age: Exploring a Virtual Sense of Geographic Place through Online
Collaborative Media and Mixed Media. Journal of Higher Education Outreach and Engagement, 18(4), 201-231.

\section{PERFIL ACADÉMICO Y PROFESIONAL DE LOS AUTORES}

Beatriz Sandia Saldivia. Ingeniero Civil (Universidad de Los Andes - ULA, Venezuela); M.A. en Liderazgo en Tecnología Educativa (George Washington University, USA); Doctorado en Tecnología Educativa (Universidad de Las Islas Baleares, España). Profesora Titular, Facultad de Ingeniería, ULA. Áreas de investigación: Tecnología Educativa, Telemática, Tecnologías de la Información y Comunicación en la Educación y Gestión del Conocimiento.

E-mail: bsandia@ula.ve; bsandia@gmail.com

Jonás Montilva Calderón. Ingeniero de Sistemas (ULA, Venezuela); M.Sc. (Case Western Reserve University, USA); Ph.D. en Computación (University of Leeds, UK). Profesor titular, Departamento de Computación, Facultad de Ingeniería, ULA. Miembro fundador del Grupo de Investigación en Ingeniería de Datos y Conocimiento (GIDyC). Realiza su investigación actual en las áreas de: Tecnologías Digitales Emergentes en la Educación Superior, Transformación Digital, Ingeniería del Software y Gestión de Procesos de Negocios (BPM).

E-mail: jonas@ula.ve; jmontilva@gmail.com

Dirección:

Universidad de Los Andes

Facultad de Ingeniería

La Hechicera, Edif. B

Mérida, Venezuela 5101

Fecha de recepción del artículo: 29/03/2019

Fecha de aceptación del artículo: 29/06/2019

Fecha de aprobación para maquetación: 10/08/2019 